\title{
DE L'IMAGE À LA NARRATION
}

\author{
Adolphe Nysenholc ${ }^{1}$
}

Dans les arts du spectacle, le comédien classique s'adapte à l'évolution du récit, se coule dans le flux des événements, réagit aux données du réel, fait face, tant bien que mal, aux retournements de situation ou coups de théâtre. Son image est d'abord créée par le récit. Même quand le héros essaie d'infléchir le courant des choses, l'acteur qui l'incarne est au service d'une diégèse qui lui préexiste, qui l'informe. On a beau être Laurence Olivier, on sera surimpressionné d'Hamlet.

Et, à la limite, dans le cinéma de fiction, qui s'est émancipé du théâtre, on demande même souvent à ceux qu'on filme de ne pas jouer, mais de se contenter d'être ${ }^{2}$. De se laisser traverser par la narration. D'où la possibilité du recours à des acteurs non-professionnels dans le cinéma muet soviétique ou autre néo-réalisme italien... Le sens de l'image sera induit par le montage, selon l'effet Koulechov.

Celui-ci avait, on le sait, demandé à Mosjoukine, devant l'objectif, de ne rien signifier de particulier; le plan de son visage neutre fut collé à un autre montrant un lit avec une odalisque et puis à un autre avec un mort. Et tout le monde crut que l'acteur exprimait

1 Professeur à l'Université Libre de Bruxelles.

2 Chaplin n'engageait de préférence pas des comédiens, à qui, estimait-il, il devait tout désapprendre; Edna Purviance était une secrétaire à San Francisco, Virginia Cherril n'était en rien comédienne, etc... 
parfaitement dans le premier cas le désir; et dans le second, le deuil. Deux plans juxtaposés énoncent un troisième sens qui n'est impliqué dans aucun des deux séparément. Un visage inexpressif peut tout dire à l'écran : il suffit de le faire traverser de diégèse ${ }^{1}$.

\section{La star et la fable}

Mais, au cinéma, l'inverse aussi se présente assez souvent. La production fondée sur le star system a tendance à faire un film avec telle vedette -avant même d'avoir commandé le récit. Et ce dernier sera composé "sur mesure". C'est-à-dire en fonction d'une image de marque. La Metro-Goldwyn-Mayer, qui se vantait d'avoir plus d'étoiles que le firmament, était ainsi motivée à les faire rayonner. Et un récit se construisait autour de chacune d'elles comme une constellation sans cesse renouvelée.

A priori, on savait que Marilyn Monroe ${ }^{2}$ en tête d'affiche serait au hit parade du box office, comme en faisaient foi les recettes records de tous ses films. Sex symbol, elle déclenchait à chaque fois l'élaboration de tout un récit qui la mettait en évidence en tant que tel. Le scénario était une explication, un dépliement, d'elle-même... Et on n'allait pas s'installer devant un écran pour l'histoire que nous racontait le réalisateur Billy Wilder ou John Huston ou Howard Hawks,

1 C'est là toute l'école du montage-roi. (Cf. C. METZ, Essais sur la signification au cinéma, tome I, Paris, Klincksiek, 1966, p. 47-49). En parfaits disciples de Koulechov, ses adeptes croyaient dans une ciné-langue, avec la conception aberrante du plan correspondant à un mot, comme chez Vertov, Eisenstein. Certes le mot change de sens selon le contexte. Mais penser que l'on peut prélever dans le réel des images-concepts avec lesquels on pourrait composer des séquences-phrases c'était méconnaître la nature et du mot et de l'image. C'était aussi avoir une foi irraisonnée dans le pouvoir absolu du montage, c'est-à-dire un des aspects de la narration. C'était manifester pour le jeu de l'acteur un mépris fondé sur le préjugé que son jeu est contre-révolutionnaire car issu de la tradition du théâtre bourgeois. Mais avec le plan-séquence, remis à l'honneur à la fin des années trente, et qui est aux antipodes du découpage extrême, le jeu de l'acteur est forcément à nouveau valorisé. Renoir, par exemple, bien conscient et reconnaissant de tout ce qu'un acteur pouvait apporter à l'image, nourrie de toute sa vie, de sa personnalité, permettait ainsi à l'acteur de développer librement son inspiration. C'était la technique qui était au sevice du comédien, et non le contraire. On sait comment il vantait les acrobaties de son caméraman pour suivre les déplacements des personnages, qu'il se refusait par ailleurs à contraindre par des marques au sol. Les acteurs contribuaient fortement à créer l'image. C'était presque reconnaître l'acteur comme co-auteur.

2 C. Berckmans, Marilyn Monroe, Paris, L'Harmattan, 1990. 
malgré leur génie de conteur; on achètait son ticket pour voir Marilyn. La star des stars. Une des images les plus fascinantes, en ce siècle.

La figure du héros précède la fable, qui confirme l'être exceptionnel comme image.

Ce n'est donc pas l'être anonyme, interchangeable, des foules du Potemkine, ni la vedette qui s'efface devant le rôle, mais la superstar, unique, irremplaçable, et dont on se sert même de la vie pour nourrir les scénarios. Ainsi son dernier film, The Misfits (1961), sur un script d'Arthur Miller, son dernier mari, dont elle vient de se séparer, commence par un divorce et semble donc renvoyer à sa vie privée, authentifiée du fait qu'elle serait écrite par quelqu'un qui la connaît à fond. Par ailleurs, Marilyn semblait y réaliser son rêve de jeunesse ; pour son dernier film, elle joue avec Clark Gable, qui fut l'idole même de son adolescence. Le public allait voir plus qu'un personnage, ou un acteur favori. Les gens allaient communier avec un mythe vivant, vivre la vraie vie d'une déesse moderne ${ }^{1}$, leur Vénus.

Et c'est une constante dans l'histoire du cinéma. Un producteur de l'entre-deux-guerres avait confié à Charles Spaak d'adapter le roman Gueule d'amour. Mais il n'y avait pas là de quoi faire un scénario. Cela ne dérangea pas le financier. Les droits avaient été achetés d'abord et avant tout pour le titre ! Car on voulait sortir un film avec Jean Gabin'. Et le titre combiné à l" "image" de l'acteur fétiche du réalisme poétique suffisait apparemment pour faire rêver, et induire une aventure romanesque à l'écran. Jean Gabin dans Gueule d'amour -hors de toute narration- était à lui seul assez convaincant pour qu'on pût faire le pari d'un investissement (économique et libidinal). Un coup de poker sur une image... Et Spaak composa une toute autre histoire, conforme à l'idée, préjugée, du personnage.

Marilyn Monroe, demeurée plus universelle dans la conscience collective, s'est hissée au-dessus de bien des vedettes pour briller d'une lumière plus vive. Son être ne semblait finalement plus faire qu'un avec son image. Celle-ci, pourtant si travaillée d'art (maquillage durant des heures, mime, chant, danse, aux répétitions nombreuses, métier appris à l'Actor's Studio) n'était qu'un artefact. N'empêche, par-delà sa mort, elle est restée si vive. Sa sensibilité a touché davantage le coeur des foules si bien que la fiction de ses films

1 Cf. E. Morin, Les Stars, Paris, Éd. du Seuil, 1957.

2 A. BAZIN, Jean Renoir, Éd. Champs Libres, 1971, p. 169. 
a fini par se confondre avec son existence même. Comme si sa chair s'était faite image : icône.

La star est plus que la somme de ses rôles: elle dégage un imaginaire, qui transcende sa personne. Comme une figure mythologique. Son image induit des récits. Son personnage devient le héros d'un cycle, d'une geste.

Et Marilyn Monroe est peut-être morte de n'avoir plus été qu'une image, une apparence, dépossédée de sa vie privée. Elle n'a vécu que pour être une image, et elle ne serait plus désormais que cela, présente dans l'absence, un être réel imaginaire, l'image d'un récit plus vrai que nature, l'image d'une histoire fabuleuse.

\section{Le mythe et ses avatars}

Et elle rejoint là le cas des auteurs-acteurs à forte personnalité. Si Marilyn pouvait refuser un scénario, voire choisir les productions qui la mettraient le mieux en lumière, sinon suggérer des modifications qui mettraient bien en évidence sa silhouette comme sa ligne de vie, les cinéastes-interprètes, de Buster Keaton à Woody Allen, avaient l'avantage d'écrire eux-mêmes le récit susceptible d'être l'expression de leur nature et de toutes leurs potentialités.

Et je voudrais montrer, à travers trois d'entre eux, comment ils donnent d'eux-mêmes une image si marquante qu'elle peut inférer le développement narratif d'une séquence (comme Eric von Stroheim dans la Grande Illusion), voire conditionner le style de narration de tout un film (tel Orson Welles et La Soif du mal), et même impliquer le déploiement de tout un cuvre cinématographique (cf. Charles Chaplin et le cycle Charlot).

\section{L'image et la séquence}

Le scénario de Charles Spaak, pour la Grande illusion, cette fois, ignorait que Eric von Stroheim serait von Rauffenstein'. Et l'apparition saississante de ce dernier dans le film est une création de Stroheim. C'est lui qui a eu l'idée de la minerve. Or, celle-ci implique que le pilote de chasse, qui au début, on s'en souvient, a descendu en

1 Cf. le scénario original, in G. VAUGEOIS, La Grande Illusion, Balland, 1974, p. 213 et P. LEPROHON, Jean Renoir, Seghers, 1967, p. 76. 
flamme Maréchal et Boieldieu, se retrouve lui-même abattu, et même déprimé. Retournement de situation. Maréchal avait le bras cassé. von Rauffenstein a la colonne vertébrale brisée ; il est touché dans sa verticalité, dans son humanité. Il a perdu sa combativité ; engoncé dans sa prothèse, il vit sur la défensive, comme retiré dans sa coquille. Il semble encore coincé dans la carlingue de son aéroplane que l'on imagine s'être écrasé au sol. Ainsi l'accessoire du corset intègre visuellement le junker dans la trame du récit dont il explicite la ligne tragique.

Mais il implique aussi le développement narratif de la séquence. Stroheim, en portant la minerve, qui protège son tronc brisé, semble se déplacer désormais avec toute la forteresse dont il a la charge. $\mathrm{Ce}$ geôlier est lui-même prisonnier de son destin. Et il est convaincu que les autres n'échapperont pas au leur, car ils ne devraient pas pouvoir s'évader de ce château-fort, de la prison qui les enferme également. Il croit même pouvoir enfermer Boieldieu dans le carcan de son amitié, puisqu'ils partagent la même caste. De fait, il est le contraire d'une main de fer dans un gant de velours. C'est un sentimental contrarié. Mou à l'intérieur comme une tortue, c'est un tendre qui donne le change. Un faux dur. Ayant tiré sur Boieldieu en fuite (patricien qui s'est sacrifié pour couvrir l'évasion de ses deux camarades plébéiens d'origine), il lui ferme les yeux avec une émotion contenue de nounou. Stroheim, tragique, donne une image complexe, démultipliée. Ainsi corseté, sanglé dans une rigueur prussienne, qu'il caricature à l'excès, il se présente en un ersatz de chancelier de fer. C'est tout l'humour sur lui-même de Stroheim dans ce jeu presque avoué avec soi.

Il incarne ainsi plus largement la noblesse qui sera abattue finalement par la guerre 14-18, et que ses grandes bâtisses en ruines, ou aux charges trop lourdes, handicapent et ne protègent plus. Cette armure dérisoire portée comme un justaucorps semble symboliser la dernière protection de la race des chevaliers, l'aristocratie européenne dont la grande guerre aura brisé définitivement les reins.

Et Stroheim évoque même ainsi, dans une ironie douloureuse, son drame personnel, de réalisateur -lequel a construit quasi tout son univers cinématographique sur la nostalgie, non sans sarcasme, de l'empire austro-hongrois- cassé par Hollywood.

Cette image d'homme bardé, mais fragilisé, est la plus forte du film, elle y est riche en résonances, elle induit en partie le récit qui semble même être généré de ses profondeurs. Ainsi Erich von 
Stroheim tient tête à trois acteurs comme Gabin, Fresnay et Dalio ; il fait le poids à lui tout seul. Il représente le récit en condensé, en puissance, -au double sens du terme. Les autres pourront s'évader ; lui n'échappera jamais à lui-même.

Et il joue avec son mythe qu'il retourne, car dans ce film pacifiste, où il fraternise avec l'ennemi, il devient le contraire de "l'homme que vous aimeriez haïr". En don Quichotte sans illusions, dont l'armure est recouverte d'un costume chamarré, genre maréchal d'opérette de Vienne, il est le dernier à combattre pour l'esprit chevaleresque... Et dans son amour pour l'ennemi, en 1938, il était ressenti comme aussi scandaleux que dans la haine qu'il inspirait au temps du muet à travers ses rôles de noblion ignoble ou d'officier cynique de la guerre précédente...

La Grande Illusion fut déclaré par Goebbels ennemi public numéro 1, et interdit en France, lors de la veillée d'armes : de part et d'autre de la frontière, l'amitié transnationale pouvait compromettre l'esprit patriotique de l'union sacrée, et émousser la combativité des belligérants. Stroheim crève l'écran. Seulement carapaçonné, blessé dans l'âme, superbe, il ne pouvait être un modèle de héros...

\section{L'image et le film}

Mais le héros principal peut même insuffler sa propre respiration à tout le récit. La figure monstrueuse, tentaculaire, d'Orson Welles, dans la Soif du mal (Touch of evil, 1957) -où il incarne un policier dévoyé au grand cœur, mais non dépourvu de pulsion fascisante, Quinlan- semble se projeter dans l'emphase de toute la mise en oeuvre cinématographique. L'image de sa corpulence, envahissante, expression d'un esprit à tendance totalitaire, se déploierait dans la redondance du temps comme elle s'étend dans l'espace.

Lors de sa première apparition, il semble énorme, avec un ventre de baleine, chef d'enquête bousculant tout sur son passage, ventripotent (bourré de coussins), quasi omnipotent. C'est qu'on le voit sortir de sa voiture avec son corps obèse, déformé en contre-plongée, qui remplit tout l'avant-plan pris en grand angulaire -tous procédés susceptibles de gonfler outre mesure le profil avantageux du personnage. Ce corps adipeux, flasque, suant, préfigure son portrait moral de représentant de l'ordre sans conscience, ni principe, dans le prolongement du maccarthysme ainsi dénoncé par le film à travers un être qui 
ne voit pas de limite à son pouvoir. Vulgaire, sardonique, vide de tout scrupules, il fabrique des preuves pour faire aboutir ses enquêtes. Il élimine même un des témoins gênants, qu'il persécute dans sa chambre, où il lui bouche toutes les issues jusqu'à ce qu'au bout de cette longue séquence claustrophobique sans coupure, il l'étrangle. Ce détective au comportement de mafieux est un boulimique des réussites rapides. Et la boursouflure du personnage est finalement en résonance avec le style néo-baroque du film dont les plans-séquences, telle la toute première sur laquelle s'inscrit le générique, sont tournées comme des marathons à vous couper le soufflel.

Ainsi, chaque séquence semble obéir à la même grandiloquence magnanime d'écriture. L'épilogue est fort semblable au prologue. Son ami de toujours, Menzies, retourné par le rival de Quinlan, Vargas, se rend à l'évidence de la profonde corruption de celui qu'il croyait pouvoir admirer à jamais (comme Holly Martins et Harry Lime dans Le Troisième Homme). Quinlan lui avait même sauvé la vie en s'interposant, et ayant reçu la balle dans la jambe à sa place, il boite toujours. Mais Menzies, convaincu de sa malfaisance, accepte de démasquer Quinlan, par le stratagème d'une sorte de talkie-walkie. Et il l'attire à l'extérieur, l'interroge, en portant un magnétophone dissimulé, de façon à ce que Vargas puisse l'entendre à distance. Ce dernier, qui use lui-même cette fois d'une trâtrise, les suit caché, en essayant de ne pas perdre le contact avec la confession enregistrée. Leurs déambulations respectives, parallèles, superposées, et croisées, évoquent le manège du début où Vargas et sa femme et l'auto aussi piégée par une bombe se côtoient, se suivent, se précèdent, en un même ballet de chassé-croisé, jusqu'à l'explosion de l'engin. Et la promenade nocturne où ils se sont engagés développe non plus seulement une emphase visuelle mais sonore : l'écho amplifié sous la haute arche du pont finit par trahir Quinlan qui s'en rend compte trop tard -comme la jeune femme dans l'auto avait l'impression d'entendre un tic-tac suspect- entraîné inéluctablement dans la mort... Il trébuche en arrière dans les immondices, au bord du canal. Vidé de sa vie, immonde, son gros corps semble flotter sur l'eau comme un ballon crevé à la dérive, baudruche pitoyable qui sombre, au moment où l'on apprend que celui qu'il accusait sur de fausses preuves (comme dans

1 Cf. P. Bogdanovich, Moi Orson Welles, Belfond, 1993, p. 438-9. (L'interrogatoire de Sanchez représente douze pages de script tournées en une seule prise, à travers trois pièces, avec sept rôles parlants.) Voir aussi p. 315-340. 
la chasse aux sorcières) était passé aux aveux... Quinlan meurt presque innocenté, s'il n'avait pas commis entre-temps un crime pour rien, et envoyé tant d'innocents sans doute à la peine de mort... Welles crée ainsi, dans l'hyperbole, à partir d'un polar mineur, et d'un potentat local de la police, moi hypertrophié, comme Kane, Macbeth, mais presque bouffon comme Falstaff, une superbe et inquiétante épopée.

\section{L'image et l'œuvre entier}

Quant à la silhouette de Charlot, elle contient rien moins que l'oeuvre muet de Chaplin. De son image est sorti tout le cycle Charlot. Du type qu'il crée dès son deuxième film est issu tout le mythe, au sens de parabole. Le récit chaplinien (sans paroles) est la légende de son image.

Charlot, avec une jaquette trop étroite, un chapeau melon trop petit, et un pantalon et des godillots trops grands, semble aristo audessus de la ceinture, et clodo dessous ${ }^{1}$. Il porte comme la hiérarchie des classes sur son être. Et ce paradigme social va se déployer dans ses aventures, où le gentleman déchu va souvent se retrouver par quiproquo dans la haute (The Count), voire être pris lui-même pour un riche (The Idle Class, où le riche a un sosie pauvre; ou plus subtilement dans City Lights, où il est visuellement pauvre pour le spectateur mais riche imaginairement pour la fleuriste aveugle), et finir au sommet de la société à la place du dictateur.

Par ailleurs, le dessus inspire la pitié. Chaplin dit comment par un effet de mime il rapproche les épaules pour se faire plus petit en face de la grosse brute et susciter de ce fait la sympathie ${ }^{2}$ et le rire d'accueil ${ }^{3}$. Et le dessous de son être est plutôt grotesque, inspirant le rire d'exclusion. C'est le siège du coup de pied au c..., qu'il reçoit d'ailleurs dignement. Et ces deux pôles affectifs -qui s'inversent sur son corps (son petit chapeau peut être ridicule ; et ses grands panards, pitoyables)- vont se distribuer tout au long de ses films, lesquels, à partir de The Tramp (1915), exploiteront de plus en plus la veine sentimentale aussi bien que burlesque. Chaplin a la force de pouvoir

1 A. NySEnHolc, L'âge d'or du comique, Éd. de l'Université de Bruxelles, 1979, p. 200. (cf. dialectique de Charlot).

2 Charles Chaplin, "What people laugh at", American Magazine, 18 novembre 1918, trad. in P. LeProhon, Charles Chaplin, Debresse, 1957, p. 67.

3 E. DUPRÉEL, "Les problèmes sociologiques du rire" (1928), in Essais pluralistes, P.U.F., 1950, p. 41. 
conjuguer les larmes et le rire. Ainsi, aux moments clefs, ses gros plans sont des coupes d'âme où il exprime l'émotion qui l'anime : on peut lire à livre ouvert sur son visage ${ }^{1}$, et notamment quand il tombe amoureux. Et ce côté quasi mélodramatique permet l'identification au personnage, qui est plutôt mis à distance dans le comique, et notamment lorsqu'il s'éloigne en drôle sur la route finale avec sa fameuse démarche, son pantalon en accordéon et ses pieds latéralisés.

Et certes, il porte des habits d'homme. Mais, avec ses vêtements mal ajustés, c'est comme s'il avait un corps en croissance d'enfant, on dirait qu'il a grandi trop vite au-dessus de la ceinture, et qu'il devrait encore grandir au-dessous ${ }^{2}$. Et cette dialectique des âges va se retrouver dans d'autres chiasmes de ses récits, où il commence en petit père protecteur pour Edna, le gosse ou un chien, et où il finit par être sauvé par eux, quasi adopté (voir la fin du Kid; le tragique du finale de City Lights, c'est que l'aveugle guérie, grâce à lui, un vagabond ! et désillusionnée, ne va peut-être pas le prendre elle, cette fois, sous son aile...).

Et, certes, le mime fonde l'art de Chaplin. Son film est aussi silencieux que sa photo. Et s'il arrive à Charlot de sussurer un mot, le plus souvent il ne parle pas, à la limite il ne semble pas habité de paroles, pas encore... De fait, muet, il est infans, selon l'étymologie. Il est vrai qu'il fait tenir son pantalon avec une épingle de nourrice (The Vagabond ); et il en a souvent le fond mouillé, comme s'il avait des couches. Ses gags sont continuellement des levées d'inhibition, comme s'il était toujours en défaut de refoulement (Freud) ${ }^{3}$, et donc pas encore bien socialisé. Il provoque le rire de sanction (Bergson) ${ }^{4}$. Et Chaplin use de la parole, avec véhémence, contre le parlant, en écrivant un article "Against the talkies" (1928). "Le jour, dit-il, où Charlot ouvrira la bouche, il cessera d'être". Cette caractéristique fondamentale du héros qui existe la plupart du temps en-dehors du langage, voire avant, conditionne toute la stratégie narratologique de Chaplin. Chaque histoire était racontée, filmée, de manière à préserver sa mutité. Elie Faure parle dans son cas de cinémime ${ }^{5}$.

1 A. NYSENHOLC, L'Age d'or du comique, op. cit., p. 40.

2 Ibid., p. 233.

3 S. FREUD, Le Mot d'esprit en rapport avec l'inconscient (1905), Paris, Gallimard, 1969 (réimpression), p. 149 (refoulement), 179 (levée d'une inhibition).

4 H. BERgSON, Le rire ou Essai sur la signification du comique, Paris, P.U.F.,1967, p. 16 (châtiment), 103 (brimade sociale), 150 (correction).

5 E. FAure, Fonction du cinéma, Gonthier, 1953, p. 35 
Charlot est continuellement double. Man-child, synthèse sociale, tragico-comique, innocent/amoral... Il est un composé de contradictions, qui constituent la dynamique de ses gags, et même le moteur de chacun de ses films.

Chaplin a ainsi confectionné pour son héros des scénarios qui semblaient émaner de son être même. Sa course en zigzag figure en modèle réduit la trajectoire de tout un récit, parcours par essais et erreurs à travers un milieu où il flanque la pagaille jusqu'à ce qu'il s'échappe par la tangente...

Chaplin crée une homologie, on ne peut plus intégrée, entre le personnage et son récit. Charlot démonte les roues d'un réveil qu'il opère comme un cœur (dans The Pawnshop) de la même façon que Chaplin met en oeuvre les rouages de toute une usine conçue comme une grande montre dans laquelle tombe son héros (Modern Times). Et si Charlot manipule une marionnette qu'il a fabriquée sous nos yeux pour la danse des petits pains, Chaplin, éminence grise de son film, manœuvre comme un théâtre de guignol la cabane en bascule sur le précipice, où s'agite Charlot comme un pantin (The Gold Rush)... Le créateur, en grand imagier, transforme les choses et les êtres par métaphore tout comme sa créature, et vice-versa. La narration est sans cesse, d'une manière ou l'autre, l'écho temporalisé de son image.

Cette homothétie esthétique ${ }^{1}$ est rare, sinon unique. D'où la forte cohérence de son mythe, et la grande résonance dans le siècle de son œuvre muette, comme parole. Charlot, porte-parole de son temps.

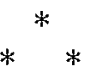

En somme, art de l'image, le cinéma, comme photographie animée, met en mouvement une image fondamentale, qui se développe en récit. Et, dans le meilleur des cas, un récit peut se montrer

\footnotetext{
1 A. Nysenholc, Charles Chaplin ou la légende des images, Paris, Méridiens Klincksiek, 1987, p. 90-92. Buster Keaton réalise un même isomorphisme entre les formes du héros et les structures du scénario, dans The General, où il était maître absolu de son produit. Il y développe un parallélisme fort entre son allure géométrisée, volontaire, dynamique, et le parcours ferroviaire de la poursuite égrenée de gags mathématiques et autres trouvailles, dont il avait également le génie. Et lui qui est si actif, et même acrobatique, il est aussi, comme la plupart des burlesques, un rêveur. Aussi, c'est tout naturellement qu'il se projettera dans l'écran de rêves pour y vivre par compensation ses amours ainsi réalisées le temps d'un songe (Sherlock Jr).
} 
peu à peu révélateur de toutes les virtualités de cette image initiale. Un récit peut être la projection anamorphosée, en devenir, d'une image originaire, qui apparaît au fur et à mesure dans le temps, comme une photo dans un bain de révélation. L'image originale contiendrait tous les éléments, comme un programme génétique à réaliser. Ce serait alors la petite forme qui engendrerait la grande. Chaplin a d'abord créé son héros, plein de possibilités, qu'il a sauvé ensuite en l'intégrant dans des scénarios susceptibles de le montrer toujours tel qu'en lui-même... L'image fondatrice peut ainsi être une construction en abyme du récit. Ce dernier fonctionnerait comme un pantographe capable de transposer l'image spatiale première sur le plan temporel... Une telle image serait la matrice de la narration. Il se réalise ainsi entre le héros et son destin, une harmonie imitative, qui tend à réduire l'arbitraire de sa vie, et qui contribue même à conférer à sa destinée davantage de sens. S'accomplit, de cette manière aussi, ce que Roman Jakobson ${ }^{1}$ appelait la fonction poétique ${ }^{2}$.

1 R. JAKOBSON, Essais de linguistique générale, Éd. de Minuit, 1956, p. 218-220.

2 N.B. Dans la modernité, l'image générique semble souvent être relativement abstraite, comme la "figure", chez Tarkovski, source d'inspiration quasi mystique, préfiguration d'où sort tout son film; ou peut-être tel archétype chez Pasolini. Chez André Delvaux, ce qui déclenche la dynamique créatrice du film est la "forme" (au sens musical), par exemple celles du rondo ou de la sonate, qui sont les principes du montage respectivement de Rendez-vous à Bray ou de Benvenuta, dont les.héros, masculin et féminin, sont pianistes. De l'image à la narration, autrement que dans le film classique, le cinéma de l'image-temps peut ainsi vibrer autant en harmoniques inouïes. 